\title{
A Practical Student Model for a Location-Aware and Context-Sensitive Personalized Adaptive Learning System
}

\author{
Guangbing Yang, Kinshuk, and Sabine Graf \\ School of Computing and Information, Athabasca University, Canada \\ \{guangbing,kinshuk,sabineg\}@athabascau.ca
}

\begin{abstract}
Student model is an essential component in any adaptive learning environment. Various student modelling approaches have been suggested in the literature that focus on learners' competencies, preferences and other personal attributes. However, there is a lack of a comprehensive student model that combines various aspects of student modelling while also considering the aspects of location and context awareness that are important for emerging ubiquitous learning environments. This paper proposes one such student model in the context of a personalized adaptive learning system, namely Personalized Adaptive Learning Dashboard (PALD), a compound student model bringing together various features of the overlay, fault, and stereotype models to provide a generic approach to modeling student with specific domain related knowledge.
\end{abstract}

Keywordst; student model; personalization; adaptation; knowledge; student modeling

\section{INTRODUCTION}

Learning Management Systems (LMS) provide a wellstructured environment supporting interaction between learner and content, learner and tutor, and among learners. However, each learner has individual characteristics, such as knowledge, skills, experience, goals, interests, learning styles, background, and so on. As a result, there is an emergent demand for tailoring learning context and content for each individual learner. Specifically, a personalized adaptive learning environment is required to acquire, organize, personalize, share and use the knowledge embedded in the learning content, and to achieve interoperability between heterogeneous information resources and services.

According to [10], personalization is a key issue in the adaptive learning environments, and it relies on the student modeling. The student model represents student in the system. Imagine the student model as an avatar of a real student in the virtual world, the dimensions of the student model correspond to the aspects of the physical student and the properties of the student model represent the characteristics of the real student. In this context, the student model can be defined as the description of student's characteristics. The process that collects information to build and update the student model is called student modeling. In the learning environments, every student exhibits unique individual characteristics and preferences. Therefore, the student model, as the representative of the student, is expected to reflect these differences. However, only student model itself cannot complete the expression of the differences. An environment needs to exist to provide personalization during the learning process. In this learning environment, the personalization performs the adaptation by deploying appropriate adaptation algorithms and suitable data structures to represent the learner's characteristics and problem-solving states [10].

[13] noted that a learning environment should start adapting to the way the learner likes to communicate and organize information as soon as the learner starts interacting with it. This requires a suitable student model developed to trace learner's learning history [10].

Some student models are built for recognizing student's cognitive patterns, some are built for evaluating student performance or problem solving skills, and some of them are created for constructing student knowledge and concept tree, like Columate, the user model server described by [5]. However, in order to carry out the personalization efficiently, the student model needs to consider both domain dependent and domain independent characteristics.

In past, various types of student models have been reported in the literature to address the personalization and adaptivity issues. The overlay model and stereotype model have been the most commonly used categories of student model.

The overlay student model focuses on the comparison between the student knowledge and the domain knowledge. It is the subset of the domain model. The student model is domain dependent and relies on domain knowledge structure. Some models are similar to overlay model, such as differential model, fault model, and perturbation model.

A stereotype model represents a set of learner's frequent characteristics [14]. New learners in this model are classified according to their initial features; each classifier is a stereotype of the student model. A significant problem in this student model emanates from the assigned initial values to the model. If the initialization is done by students' self-descriptions, such as pre-test or questionnaires, the initialized model may not be very precise and accurate against specific domain knowledge. As a result, this model may take a long time to adjust and calibrate, and hence frustrate the student and may cause a drop in learning motivation.

The student model proposed in this research is a compound model, bringing together various features of the overlay, fault, and stereotype models to provide a generic approach to modeling student with specific domain related knowledge. This way, the model not only can exhibit unique individual characteristics and preferences of each learner by monitoring and tracing the changes of their knowledge, skills, interests, but can also classify the learners according to their performance, individual learning behaviors and activities. It compares the current profile of the learner with historical one to acquire the real improvements that learners have gained during a learning 
session. The proposed student model is described in this paper in the context of a personalized adaptive learning system, namely Personalized Adaptive Learning Dashboard (PALD).

The rest of this paper is structured as follows. In Section 2, the PALD is introduced and discussed in the context of the requirements to build the student model. Section 3 discusses the dimensions of the student model and characteristics of the students included in the model. In section 4, an overview of the application implementation is discussed, followed by a simple experiment with the PALD for evaluating the student model. The paper concludes with a summary of outcomes and discussion on future work.

\section{Personalized AdAPTIVE Learning DashboARD}

The Personalized Adaptive Learning Dashboard (PALD) is an adaptive learning environment supported by Web 2.0 technologies. Over the past two decades, lots of efforts have been put into exploring and researching the benefits of adaptivity in e-learning, and thereby, a number of research projects and systems already use adaptivity [11]. However, majority of these systems do not support the personalization and adaptivity at the same time. For example, AHA [8] and AHAM [9] are adaptive e-learning systems with weak personalization support. Moodle and Sakai are quite popular e-learning systems with personalization functionalities, but they do not implement the adaptivity features. ALFANET [15] and NetCoach [16] are two systems supporting both adaptivity and personalization functionalities, but they lack appropriate social collaboration and multimedia content support. PALD is designed and developed as a personalized adaptive learning system that supports functionalities of adaptivity, personalization, and social collaboration. Furthermore, the PALD is a context-aware system. It supports mobile learning as well as e-learning. It supports the location awareness and activity recognition in order to provide the efficient adaptivity and personalization.

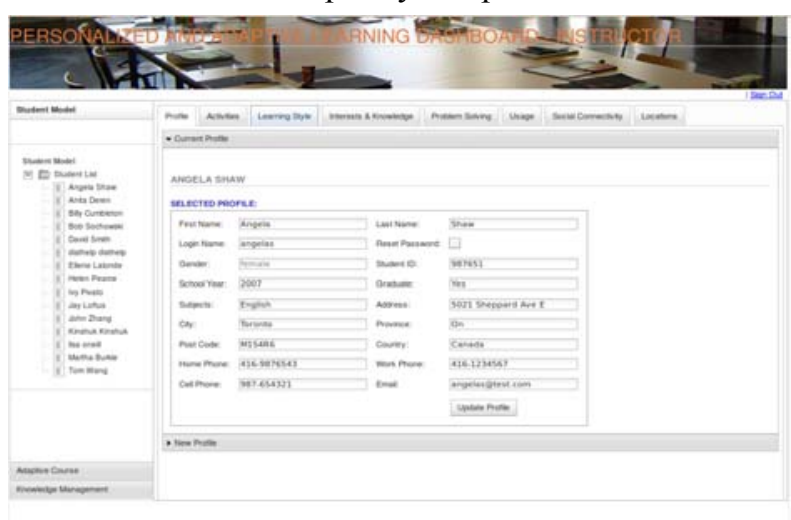

Figure 1. The student profile interface of PALD

Figure 1 shows the main interface of PALD. The system visualizes the student information and provides an adaptive learning environment for educators for managing, monitoring, and tracing the learning activities and learning progress. It also provides a platform for knowledge management and course adaptivity.

From the design point of view, there are several facts about PALD that influence the construction of the student model:1) PALD constructs student's knowledge tree that is domain dependent model overlaying the student model for competencies; 2) it contains information about student's learning performance, a factor used in pedagogical decision making; 3) it supports location-awareness, context-sensitiveness and social collaboration factors which influence adaptivity and personalization; and 4) it consists of different components that offer different information that are needed by different kinds of pedagogical decision-making. These decisions in turn influence the adaptation on content level and on the presentation level [4].

\section{The CONCEPTUAL ARCHitecture OF A StUdENT MODEL IN PALD}

Figure 2 shows the dimension map of the student model to illustrate the characteristics that are taken into account in the architecture of the student model in PALD system.

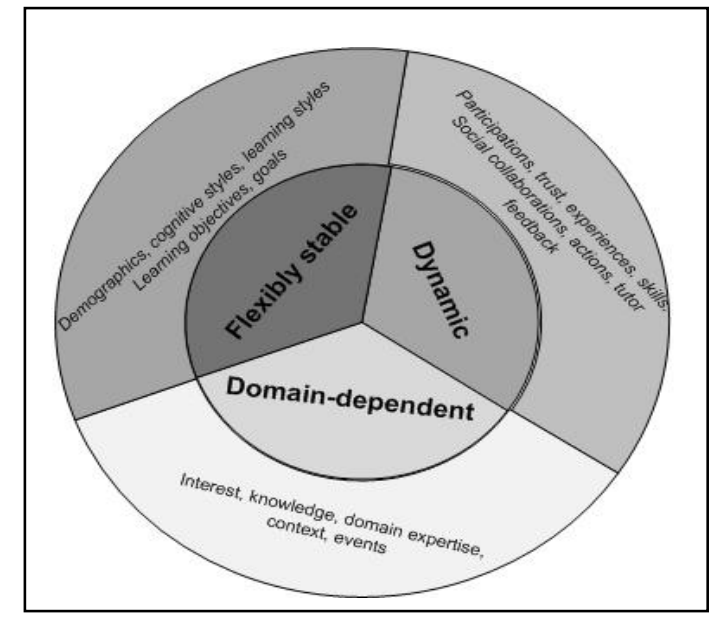

Figure 2. Dimension map of the student model in PALD

In this map, the characteristics of a student are categorized into three sectors, flexibly stable, dynamic, and domain-dependent. In flexibly stable dimension, student's demographics, cognitive styles, learning objectives and goals are the parameters to be processed during the student modeling. In the dynamic dimension, student's experiences with the learning systems, skills, social collaborations, learning actions, learning behaviors, motivations, attitudes, and tutor's feedbacks are considered in order to infer student's knowledge of concepts and learning performance in a learning session. In the domaindependent dimension, student's knowledge level in a specific domain topic, student's interests to certain topics or subjects, and events raised during learning process are considered as domain knowledge dependent parameters.

Students' knowledge level is assessed using both direct and indirect methods. In direct method, the assessment of the knowledge is carried out by asking the students questions, assessing the quizzes, observing student's online practice exercises, and so on, after a learning module is completed. In indirect method, the assessment of student's knowledge level is performed by monitoring student's activities and feedback that could indicate any problems they encountered in the use of the learning environment. For example, PALD tracks the amount and 
type of the student's activities, hours students spent in a particular learning session, the number of logons, and the number and proportion of individual student contributions to the class activities (such as discussion forum posts, comments to learning materials, and so on). All these parameters indirectly indicate learners' motivations and possible problems during the learning process [10].

Similar to the assessment of the knowledge level, student's interest to a certain topic or subject can be assessed through using the five star rating [2] method by asking the students to directly input their assessment; or assuming and estimating students' interests indirectly by monitoring students' interaction in the system, any feedback they have provided, calculating the number of times students have posted their comments regarding a particular topic to the bulletin board or discussion forum, and by calculating how many times the students have searched the same topic or subject by keywords or tags in the system.

The dynamic parameters are collected and structured as activity trees [10]. The tree path maps the sequencing information of the learning activities (IMS SS http://www.imsglobal.org/ simplesequencing/). The tree nodes indicate the learning activities and the relationships between each learning activity are identified by the hierarchy of the tree. [10] explained that the sequencing information should incorporate rules that described the branching or flow of learning activities through the learning content according to the outcomes of the learner's interactions with the content. In addition to the definition of the information sequencing, the IMS SS also defines some simple sequencing behaviors, such as navigation, flow, sequencing, termination, delivery, exit, selection, and rollup processes, etc. Each activity associates with a set of sequencing behaviors. In our student model, the learning activities are formed as trees and the processes of the sequencing behaviors traverse the trees to apply the sequencing rules, which are defined by IMS SS, and identify the activities and their associated learning contents. The results of the identification of the activities and their associated learning content create desired learning experiences for the student. Furthermore, these learning experiences influence student's knowledge level, learning performance, goals, etc. Through validating these learning experiences, the student model is updated.

\section{STUdent Modeling IN PALD}

The description above gives a brief conceptual structure of the student model implemented in the PALD and what parameters are involved in the student modeling, but does not suggest how PALD performs the modeling process, especially the inference mechanism that is implemented in the student model to carry out the adaptation operations.

The process of student modeling in the PALD is as follows. It receives reports of student's learning activities from components that are either integrated with PALD or as external applications. The reported activities include contents the student have read, questions answered, links followed, discussion forums joined, social networks joined, and components visited (as permitted for observation by the students). From these reported activities, the PALD infers student parameters introduced in the previous section. Inference is done using various approaches, ranging from simple ad hoc match to Bayesian Network [6, 12] and ontology reasoning [7].

After the inference operation, the student model is validated and updated by estimating the knowledge about different topics from the learning materials the student is supposed to learn. The question that rises here is how to associate the knowledge concepts with learning materials. There are two spaces involved in this association. One space is called Knowledge space which is the network of knowledge concepts. Another space is called Hypermedia space which is the network of hypertext pages with learning contents [5]. Indexing is a common approach to associate knowledge concepts with educational materials. There are several ways for the indexing approach [5]. In our design, the fragment indexing approach is implemented to map the knowledge concepts with fragments of contents in the learning material. Typically, the fragments of contents in the learning materials are marked up with metadata implemented in Learning object metadata (LOM). The hierarchy relationships among fragments are defined by LOM. The connections among knowledge concepts in the knowledge space and the content fragments in the hypermedia space make it possible to map the hierarchy relationships of the fragments of learning contents to the hierarchy relationships of the knowledge concepts. Other words, through the connections, by knowing the relationships among content fragments, the relationships among the knowledge concepts can also be known. This deduction also exists reversely. The relationships among content fragments are defined by LOM, which is already known by the system. By monitoring the student's learning activities the path of the access of the content fragments can be indicated. Through the connections between knowledge space and hypermedia space, the path of the knowledge concepts can be identified for a particular student. This identification is forwarded to the pedagogical model and domain model in the PALD to decide which pedagogical rules will be involved and assume which knowledge level the student has achieved after the knowledge assessment process which is usually carried out by asking questions, doing exercises and undertaking quizzes. As described in the previous section, the student model updates are based on the changes in the knowledge level and other parameters such as performance and learning goals.

Besides the inference of learner's knowledge of concepts, the interest, learning styles, performance, and other parameters in the student model are also inferred. The knowledge inference is implemented using Bayesian Networks [12] or machine learning methods. The interest and social collaboration adaptation can be implemented using information retrieval methods, such as natural language processing or probabilistic methods.

One important thing that has not been mentioned yet is the initialization of the student model. When a new student registers into the system, a new student model is established for him/her. However, the question that rises here is how the system can determine the knowledge level for a new student. [1] summarized three approaches to initialize the student model. The student model described in this paper is a hybrid model, which is a combination of an overlay model and a stereotype model. Thus, the 
initialization of the student model is carried out by the stereotype partition.

\section{AN OVERVIEW OF THE IMPLEMENTATION OF THE STUDENT MODEL}

The student model is the main component in the PALD system. The PALD is an adaptive learning environment supported by Web 2.0 technologies and service-oriented architecture. It has been designed and developed as a J2EE application. As a service layer, the student model has been developed as a Java API with a service layer embedded to facilitate the process of the student information acquisition.

As described before, the entire PALD consists of several components. From the system and software architecture point of view, the student model is treated as a separated component that interacts with other components through Web services. The inputs of the student model are XML format data from various components. The results of the processing are stored in a student profile as an XML format document. In this XML document, a set of values that indicates student's current knowledge level, demographic information, learning style, and interest, etc., is indicated.

The student model can be accessed directly by invoking functions of web services or in a synchronized replicated way which means each component can keep its own student data as a snapshot of the main student model.

\section{CONCLUSIONS}

The process of arranging personalized adaptive learning experiences is a very complex one [3]. The complexity of this problem comes from the difficulty to identify student's characteristics that influence student's learning experiences in the personalized adaptive learning environment. The further complexity comes from the classification of the student characteristics to various categories of the parameters of the student model. Each category of the parameters of the student model is processed differently. The next complexity comes from the difficulty to map knowledge concepts to the fragments of the learning contents and then to find the path of an efficient way to associate the knowledge concepts with the learning materials. Without identification of the student knowledge level in certain domain knowledge, the system has difficulty to make pedagogical decisions and has difficulty to provide suitable learning materials to the student. Herein, there is no doubt that the student model has extremely important role in the personalized adaptive learning system. Many researchers have already done lots of excellent works in the area of student model and student modeling. However, similar research efforts will continue because the new technologies and new methodologies are being invented continually.

In further work, we will continue the development of the student model and design and develop an approach to fine tune the parameters used in the student modeling. We also plan to involve the student group concept to facilitate the student model initialization process. In addition, further research with semantic based distribution services derived from the adaptive context and reuse of personalization functionalities against the requirements of a mobile environment will be conducted. As a result, we plan to extend our prototype application and investigate semantic based web services to support the mobile environments to facilitate the context-aware approaches.

\section{ACKNOWLEDGMENT}

This study is supported in part by the NSERC, iCORE, Xerox, and the research related funding by Mr. A. Markin.

\section{REFERENCES}

[1] Aïmeur, E., Brassard, G., Dufort, H., \& Gamps, S. (2002). CLARISSE: a machine learning tool to initialize student models. Lecture Notes in Computer Science, 2363, 718-728.

[2] Akpinar, Y. (2008). Validation of a Learning Object Review Instrument: Relationship between Ratings of Learning Objects and Actual Learning Outcomes. Interdisciplinary Journal of ELearning and Learning Objects. Vol 4, 2008.

[3] Aroyo, L., Dolog, P., Houben, G.-J., Kravcik, M., Naeve, A., Nilsson, M. \& Wild, F. (2006). Interoperability in Personalized Adaptive Learning. Educational Technology \& Society, 9 (2), 4-18.

[4] Beaumont, I., \& Brusilovsky, P. (1995). Educational applications of adaptive hypermedia. In K. Nordby, P. Helmersen, D. J. Gilmore, \& S. A. Arnesen (Eds.), Human-Computer Interaction, London: Chapman \& Hall, 410-414.

[5] Brusilovsky, P. (2003). Developing adaptive educational hypermedia systems: From design models to authoring tools. In T. Murray, S. Blessing \& S. Ainsworth (Eds.), Authoring Tools for Advanced Technology Learning Environment, Dordrecht: Kluwer, 377-409.

[6] Bunt, A., \& Conati, C. (2003). Probabilistic student modeling to improve exploratory behaviour. User modeling and User Adapted Interaction 13, 269-309.

[7] Conlan, O., O'Keeffe, I., \& Tallon, S. (2006). Combining adaptive hypermedia techniques and ontology reasoning to produce dynamic personalized news services. Lecture Notes in Computer Science, 4018, 81-90.

[8] De Bra, P. \& Calvi, L., (1998). AHA! An open Adaptive Hypermedia Architecture. The New Review of Hypermedia and Multimedia, vol. 4, pp. 115-139, Taylor Graham Publishers, 1998.

[9] De Bra, P., Aerts, A., Smits, D. \& Stash, N. (2002). AHA! meets AHAM. Second International Conference on Adaptive Hypermedia and Adaptive Web-Based Systems, May 2002. Springer LNCS 2347, pp. 381-384.

[10] Devedzic, V. (2006). Semantic Web and Education, New York: Springer.

[11] Hauger, D \& Kock, M. (2007). State of the art of adaptivity in elearning platforms. In LWA 2007: Lernen - Wissen - Adaption, Halle, WorkshopProceedings, pages 355-360, 2007.

[12] Millan, E., Agosta, J.M., \& Perez de la Cruz, J. L. (2001). A Bayesian Student Modeling and the Problem of Parameter Specification. British Journal of Educational Technology, 32, 171181

[13] Pednault, E.P.D., (2000), Representation is everything, Communications of The ACM 43(3):80-83.

[14] Rich, E. (1979). User Modeling via Stereotypes. Cognitive Science $3,329-354$

[15] Santos, O.C., Boticario, J.G. \& Barrera, C. (2005). aLFanet: An adaptive and standard-based learning environment built upon dotLRN and other open source developments. Foro hispano de .LRN y software libre educativo. Retrieved 26 March 2009 at: http://www.ia.uned.es/ jgb/publica/dotrln-ocsjgbcb-final.pdf

[16] Weber, G. \& Brusilovsky, P. (2001). ELM-ART: An adaptive versatile system for Web-based instruction, International Journal of Artificial Intelligence in Education, 12 (4), 351-384. 